\title{
Ultra-magnifying narrow-band imaging for endoscopic diagnosis of gastric intestinal metaplasia: a pilot image analysis study $D$
}

\section{다(1) $(2)$}

Authors

Hiroyoshi Iwagami ${ }^{1}$, Noriya Uedo ${ }^{1}$, Hon-Chi Yip ${ }^{2}$, Satoki Shichijo', Takashi Kanesaka ${ }^{1}$, Akira Maekawa ${ }^{1}$, Sachiko Yamamoto $^{1}$, Koji Higashino', Yoji Takeuchi ${ }^{1}$, Ryu Ishihara ${ }^{1}$, Shin-ichi Nakatsuka ${ }^{3}$

Institutions

1 Department of Gastrointestinal Oncology, Osaka International Cancer Institute, Osaka, Japan

2 Division of Upper Gastro-intestinal and Metabolic Surgery, Department of Surgery, Prince of Wales Hospital, The Chinese University of Hong Kong, Hong Kong, China

3 Department of Diagnostic Pathology and Cytology, Osaka International Cancer Institute, Osaka, Japan

submitted 10.5.2020

accepted after revision $\quad 24.11 .2020$

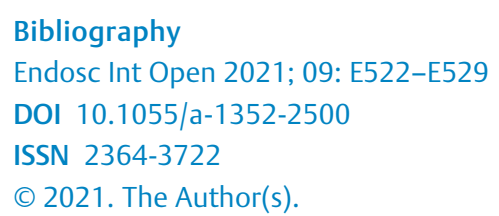

This is an open access article published by Thieme under the terms of the Creative Commons Attribution-NonDerivative-NonCommercial License, permitting copying and reproduction so long as the original work is given appropriate credit. Contents may not be used for commercial purposes, or adapted, remixed, transformed or built upon. (https://creativecommons.org/licenses/by-nc-nd/4.0/)

Georg Thieme Verlag KG, Rüdigerstraße 14,

70469 Stuttgart, Germany

Corresponding author

Noriya Uedo, Department of Gastrointestinal Oncology,

Osaka International Cancer Institute, 3-1-69 Otemae,

Chuo-ku, Osaka 541-8567, Japan

Fax: +81-6-6945-1900

noriya.uedo@gmail.com

\section{ABSTRACT}

Background and study aims Narrow-band imaging (NBI) with or without magnification has recently been used for diagnosis of gastric intestinal metaplasia (GIM). Endocytoscopy is a newly developed endoscopic technique that enables ultra-high $(500 \times)$ magnification of the digestive tract mucosa. This study aimed to analyze the ultra-magnifying NBI characteristics of GIM.

Patients and methods This was a retrospective observational study conducted in a cancer referral center. Patients who underwent ultra-magnifying NBI of the gastric mucosa using endocytoscopy were eligible. A soft black cap was used for non-contact observation. We compared the characteristic findings of GIM by ultra-magnifying NBI of metaplastic and non-metaplastic mucosae. A reference standard for GIM in this study was conventional magnifying NBI findings of GIM.

Results We obtained 28 images of metaplastic mucosa and 32 of non-metaplastic mucosa from 38 patients. Ultramagnifying NBI revealed the cobblestone-like cellular structure in the marginal crypt epithelium of metaplastic and non-metaplastic mucosa. Diagnostic values (sensitivity, specificity, accuracy and kappa value [95\% confidence interval]) for the heterogeneous cellular structure and rough contour of the marginal crypt epithelium were $82 \%$ (68\%$96 \%), 94 \%(85 \%-100 \%), 88 \%(80 \%-96 \%)$, and 0.70 , and $86 \%$ (73\%-99\%), $94 \%(85 \%-100 \%), 90 \%(82 \%-98 \%)$, and 0.71 , respectively.

Conclusions The characteristic ultrastructural features of GIM were identified by ultra-magnifying NBI, warranting validation of diagnostic value in a prospective study.

\section{Introduction}

Gastric intestinal metaplasia (GIM) is a high-risk mucosal condition for development of gastric cancer; therefore, individuals with GIM are recommended to undergo surveillance endoscopy $[1,2]$. Currently, the standard method for diagnosing GIM is histological examination of biopsy specimens $[3,4]$. However, vir- tual chromoendoscopy including narrow-band imaging (NBI), with or without magnification, has recently been used for identification of patients at high risk of development of gastric cancer [2, 5-7].

Endocytoscopy is a newly developed endoscopic technique that provides ultra-high $(500 \times)$ magnification of the digestive tract mucosa [8]. When we performed observation of the gas- 
tric mucosa using a cap, we noticed that endocytoscopy showed ultra-magnifying reflected light images of the epithelium using NBI short wavelength light. The aim of this preliminary image analysis was to explore using endocytoscopy the characteristic ultra-magnifying NBI findings of GIM.

\section{Patients and methods}

\section{Study design and participants}

This was a retrospective observational study conducted in a cancer referral center, Osaka International Cancer Institute, Japan. Patients who underwent esophagogastroduodenoscopy using endocytoscopy between January and May 2019 were eligible. We excluded patients with unevaluable images of the mucosa, that is, mucosa covered with mucus or blood, and out-of-focus mucosal images. All patients gave written informed consent for the endoscopic procedure and for the use of endoscopic images for clinical studies, and were provided the opportunity to opt out from this study. All clinical data and endoscopic images were anonymized for analysis. The study protocol was approved by the Institutional Review Board on 3 June 2019 (No. 19044).

\section{Endoscopic procedure}

All examinations were performed by an endoscopist (N.U.) who had >10 years' experience in performing magnifying NBI. Just before the examination, the patients ingested a mixture consisting of a mucolytic agent (20,000 U pronase, Pronase MS; Kaken Pharmaceutical Co. Ltd., Tokyo, Japan), defoaming agent (80 mg, 2 \% dimethicone, GASCON Drops; Kissei Pharmaceutical Co. Ltd., Matsumoto, Japan), and $1 \mathrm{~g}$ of sodium bicarbonate diluted in $40 \mathrm{~mL}$ of water. Topical anesthesia was performed with anesthetizing gel (200 mg $2 \%$ lidocaine, xylocaine viscous; Aspen Japan K.K., Tokyo, Japan) a few minutes before insertion of the endoscope. Conscious sedation was given with intravenous midazolam (2.5-5.0 mg, Dormicum; Maruishi Pharmaceutical Co. Ltd. Osaka, Japan).

The endocytoscopy system consisted of an ultra-high magnification zoom videoendoscope (EVIS-H290EC; Olympus Co. Ltd., Tokyo, Japan), light source (CLV-290; Olympus), and image processor (CV-290; Olympus). A soft black cap (MAJ-1989; Olympus) was mounted on the tip of the endoscope. For noncontact observation, working distance was adjusted by the protruding length of the cap, to make the long dimension of the endoscopic image $\sim 2 \mathrm{~mm}$ ( Fig. 1). Structural enhancement was set at B8 for NBI. Brightness control was set at average mode.

As standard clinical practice, the background gastric mucosa was observed by NBI with or without magnification to assess gastric cancer risk $[6,7]$. After conventional magnifying NBI of the gastric mucosa, ultra-magnifying NBI was performed on the same area to obtain corresponding video images ( $\triangleright$ Video 1). The maximum magnification level of the endocytoscope was optimized for contact observation; therefore, the endoscopist had to adjust the magnification by fine movement of a lever to focus the endoscopic images during non-contact ultra-magnifying NBI. Water immersion using a scope insuffla-

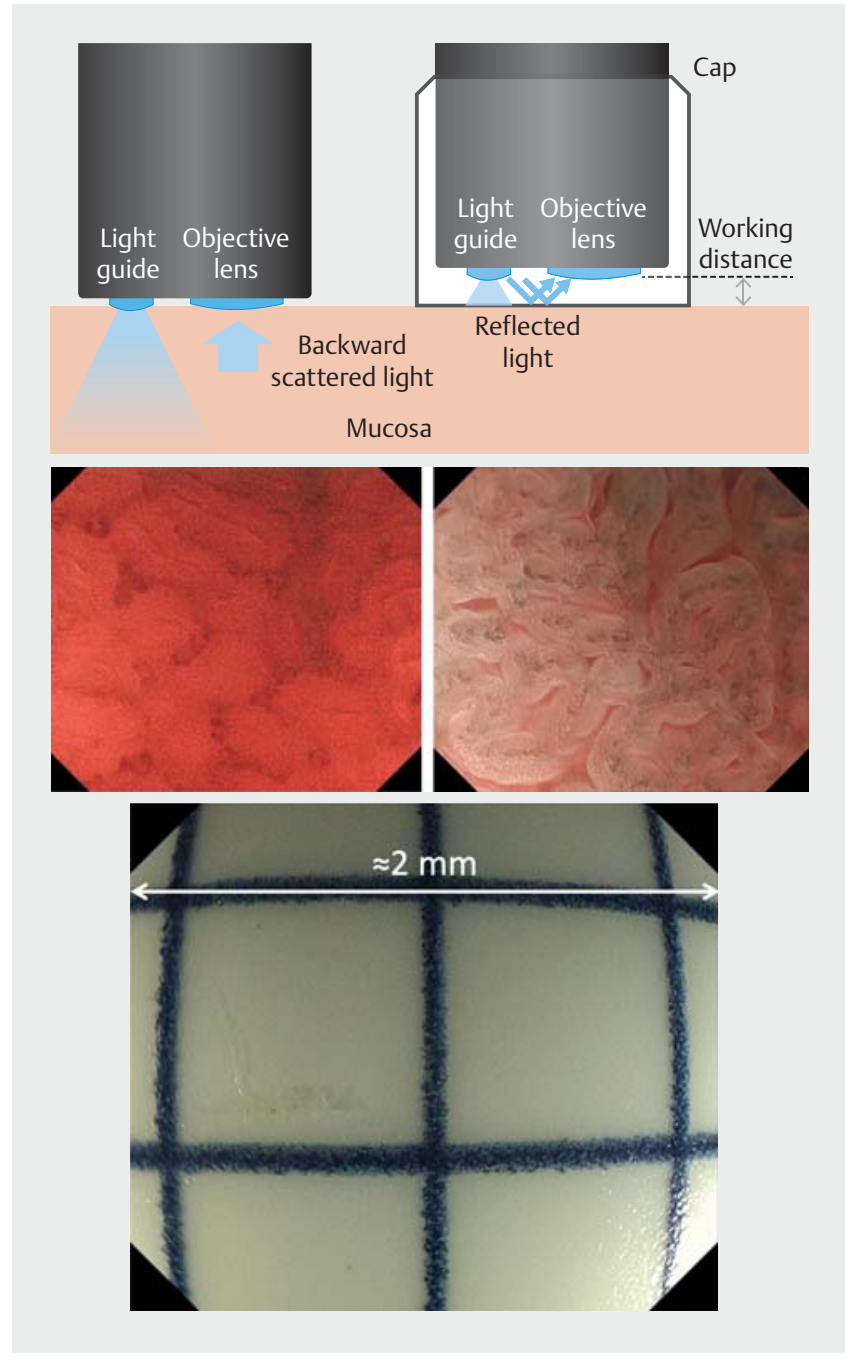

- Fig. 1 Difference between contact and non-contact observation. A silhouette image of backward scattered light was seen under contact observation, while reflected images of the mucosa were seen under non-contact observation. The working distance was adjusted by projection length of the cap to make the long dimension of the endoscopic image $\sim 2 \mathrm{~mm}$.

tion button or water pump (OFP-2; Olympus) was often used to avoid catching the light at the mucosal surface in endoscopic images and to achieve a natural magnification effect [9]. Air insufflation and the water pump were set at the lowest pressure. All images were stored in the computerized image server (SolemioEndo; Olympus). In some patients, ultra-magnifying NBI was performed on the areas from where biopsy specimens were taken, according to the updated Sydney system, to compare endocytoscopic and histological findings.

\section{Endoscopic images and definitions of GIM}

The endoscopic findings of GIM were regarded as a reference standard for GIM. The metaplastic mucosa was defined as having any indicative endoscopic signs of GIM; that is, light blue crest (LBC) [5], marginal turbid band [10], or white opaque substance (WOS) [11] in conventional magnifying NBI. Non-meta- 


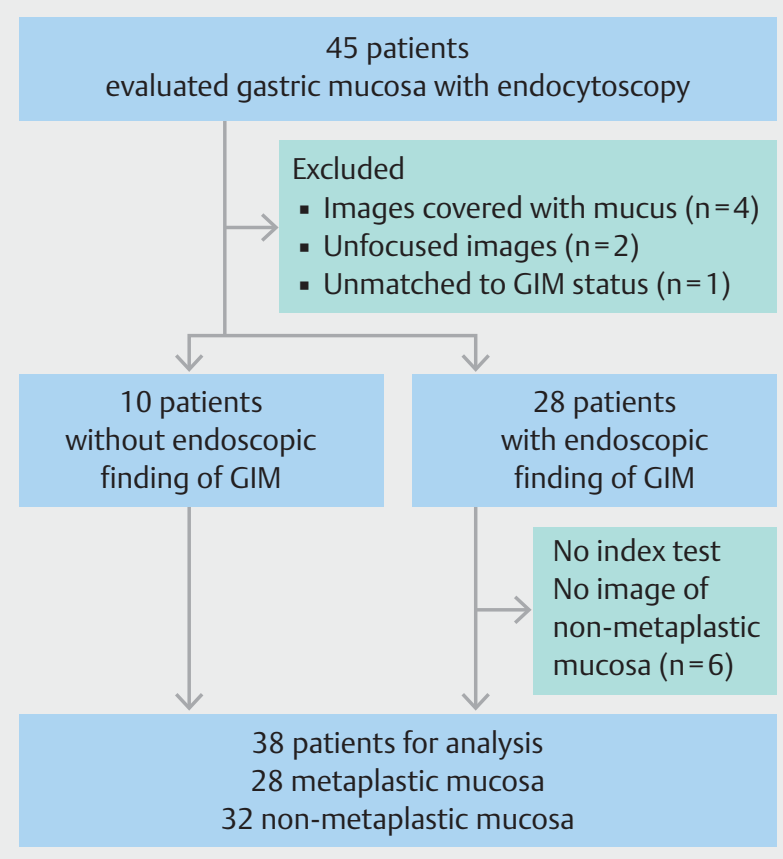

Fig.2 Flowchart of study subjects.

plastic mucosa was defined as having none of the above-mentioned characteristic signs of GIM. When multiple areas were observed in the same patient, only images of the most representative area were chosen for analysis. In patients with endoscopic findings of GIM, images of one area of metaplastic and non-metaplastic mucosae were used for analysis. In patients without endoscopic findings of GIM, images of one area of non-metaplastic mucosa were used.

\section{Helicobacter pylori infection status}

Helicobacter pylori infection was diagnosed based on antibody titer and histological examination. When anti-H. pylori antibody titer was $<3 \mathrm{U} / \mathrm{mL}$ and no $H$. pylori-like organism was found in biopsy specimens, the patient was considered to be uninfected. When anti-H. pylori antibody titer was 3 to $9 \mathrm{U} / \mathrm{mL}$ and no $H$. pylori-like organism was found in biopsy specimens, the patient was considered to have had previous $H$. pylori infection. When patients had either anti- $H$. pylori antibody titer $\geq 10 \mathrm{U} / \mathrm{mL}$ or $H$. pylori-like organisms in biopsy specimens, the patient was considered to have active $H$. pylori infection.

\section{Outcome and statistical method}

Measured outcomes were sensitivity, specificity, and accuracy of each characteristic ultra-magnifying NBI finding for the metaplastic mucosa. The data were presented with $95 \%$ confidence intervals (Cls). Interobserver variability for evaluation of each ultra-magnifying NBI finding between two endoscopists (H. I. and N. U.) was examined, and it was presented with kappa value [12].

\begin{tabular}{|c|c|}
\hline Patients & $\mathrm{n}=\mathbf{3 8}$ \\
\hline \multicolumn{2}{|l|}{ Sex } \\
\hline - Male & $25(66)$ \\
\hline - Female & $13(34)$ \\
\hline Median age (range) & $72(46-88)$ years \\
\hline \multicolumn{2}{|l|}{ Indication } \\
\hline - Screening endoscopy for GC & $4(11)$ \\
\hline - Pretreatment evaluation of GC & $18(47)$ \\
\hline - Surveillance after ER for early GC & $16(42)$ \\
\hline \multicolumn{2}{|l|}{ H. pylori infection status } \\
\hline - Uninfected & $1(2.6)$ \\
\hline - Past infection & $19(50)$ \\
\hline - Active infection & $9(24)$ \\
\hline - Not evaluated & $9(24)$ \\
\hline \multicolumn{2}{|l|}{ Endoscopic findings of GIM } \\
\hline - Absent & $10(26)$ \\
\hline - Present & $29(76)$ \\
\hline No. of evaluated mucosal images & $\mathrm{n}=60$ \\
\hline - Non-metaplastic mucosa & $32(53)$ \\
\hline - Antrum & $22(37)$ \\
\hline - Corpus & $10(17)$ \\
\hline - Metaplastic mucosa & $28(47)$ \\
\hline - Antrum & $21(35)$ \\
\hline - Corpus & $7(12)$ \\
\hline $\begin{array}{l}\text { Data expressed as number (\%). } \\
\text { ER, endoscopic resection; GC, gastric car } \\
\text { plasia. }\end{array}$ & tric intestinal meta- \\
\hline
\end{tabular}

\section{Results}

\section{Study subjects}

Forty-five patients underwent endoscopic examination using endocytoscopy between March and June 2019. The following patients were excluded: four whose mucosa was covered with mucus that interfered with observation of cellular structure; two with unfocused images; and one whose ultra-magnifying images could not be matched to conventional magnifying images for determination of GIM status, leaving ten patients without endoscopic findings of GIM and twenty-eight patients with endoscopic findings of GIM for enrollment. Non-metaplastic mucosa was not assessed in six patients with endoscopic findings of GIM, therefore twenty-eight images of metaplastic mucosa and 32 images of non-metaplastic mucosa were finally extracted for analysis from 38 patients ( $\triangleright$ Fig.2). Demographic of the patients and areas were listed in $>$ Table $\mathbf{1}$. 


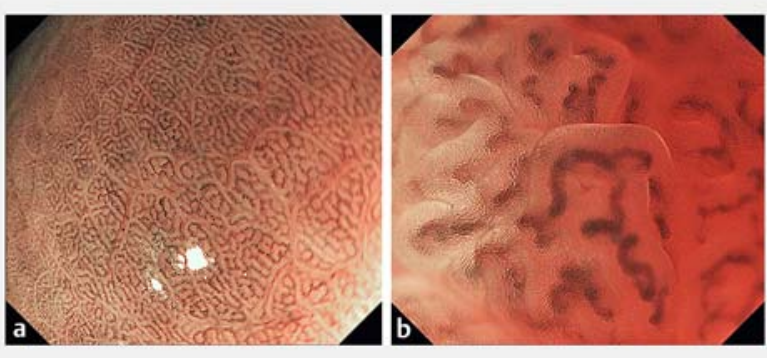

- Fig. 3 a Conventional and b ultra-magnifying NBI of non-metaplastic mucosa. NBI: narrow-band imaging.

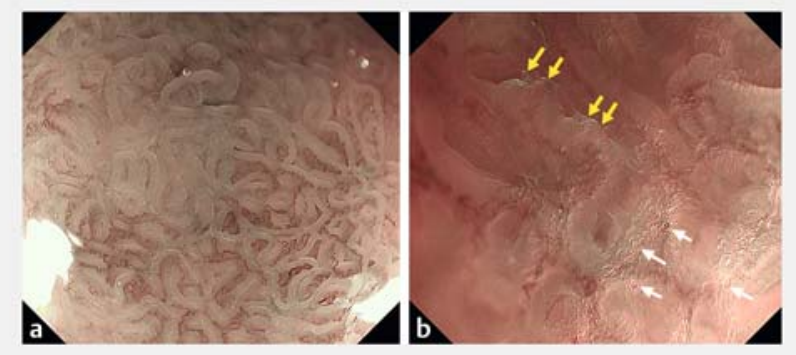

- Fig. 4 a Conventional and b ultra-magnifying NBI of metaplastic mucosa, showing LBC (yellow arrows) and sporadic large cells (white arrows). LBC, light blue crest; NBI, narrow-band imaging.

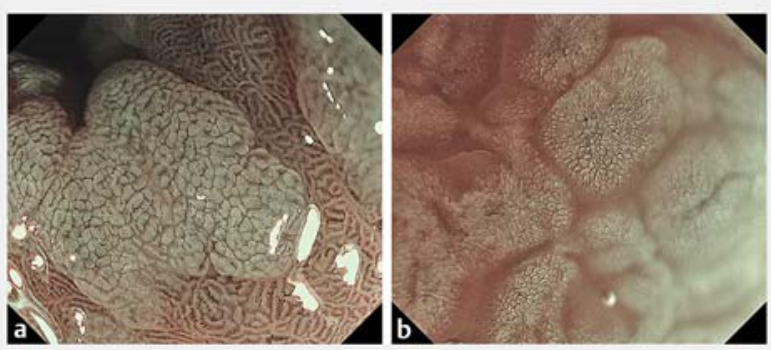

- Fig. 5 a Conventional and $\mathbf{b}$ ultra-magnifying NBI of the metaplastic mucosa with WOS. NBI: narrow-band imaging; WOS: white opaque substance.

\section{Ultra-magnifying NBI findings of metaplastic and non-metaplastic mucosa}

Ultra-magnifying NBI showed cobblestone-like appearance that consisted of small compartments partitioned by membranous septa in the marginal crypt epithelium ( Fig.3b, $>$ Fig.4b, $\checkmark$ Fig. 5b, $>$ Fig.6). We called this appearance the cellular structure.

In comparison with non-metaplastic mucosa ( $>$ Fig. 3a), we found there were characteristic ultra-magnifying NBI findings in cellular structure, contour of the marginal crypt epithelium, and presence of LBC and WOS in the metaplastic mucosa ( $\vee$ Fig.4a and $>$ Fig.5a). Heterogeneous cellular structure: in the non-metaplastic mucosa, the cellular structure was round

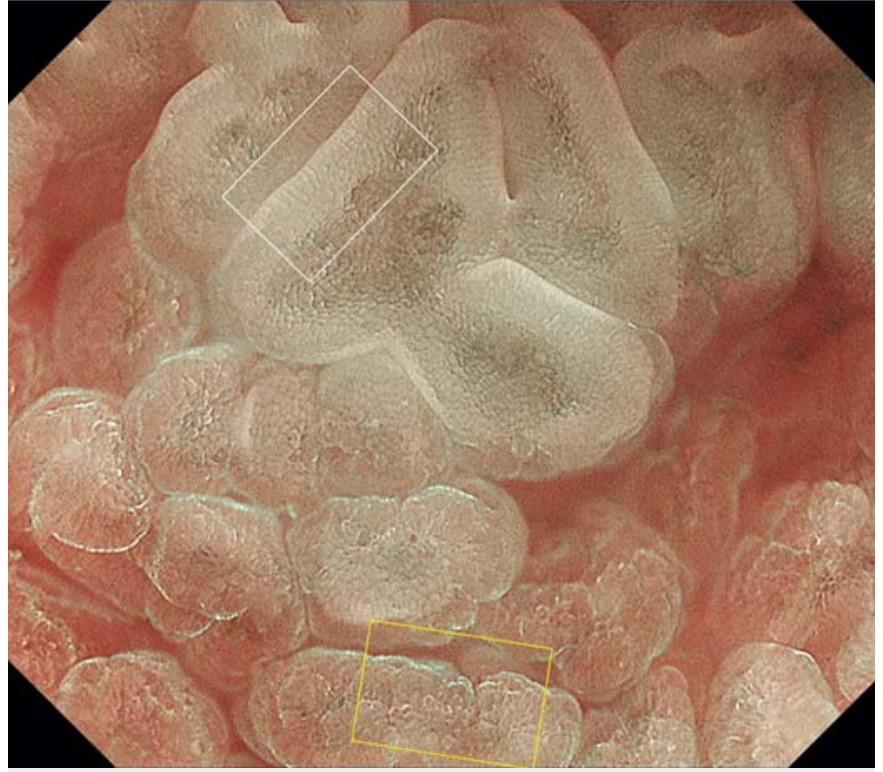

- Fig. 6 Ultra-magnifying NBI of boundary between metaplastic (lower half) and non-metaplastic mucosa (upper half). NBI, narrowband imaging. Areas in the white and yellow outlined rectangles correspond with $\triangleright$ Fig. $\mathbf{7 a}$ and $>$ Fig. $\mathbf{7 b}$, respectively.

or oval and appeared similar and homogeneously arranged like fish scales ( $>$ Fig. 3 b). In contrast, in the metaplastic mucosa, the shape, size and arrangement of the cellular structure were heterogeneous, and round cells larger than the others were often distributed sporadically in the marginal crypt epithelium ( $\triangleright$ Fig.4b). Rough contour of the marginal crypt epithelium: in the non-metaplastic mucosa, the contour of the marginal crypt epithelium was smooth and appeared as a line ( $\mathbf{F i g}$. $\mathbf{3 b}$ ). In the metaplastic mucosa, the contour of the marginal crypt epithelium was rough, and often appeared as multiple lines ( $>$ Fig. 4b). LBC: this was seen in the metaplastic mucosa but not in the non-metaplastic mucosa. Because roughness of the LBC became apparent in ultra-magnifying NBI, diagnosis of LBC was easier than that in conventional magnifying NBI. In conventional magnifying NBI, the LBC was observed only on the edge of the marginal crypt epithelium, whereas, in ultra-magnifying $\mathrm{NBI}$ images, it was observed also inside the marginal crypt epithelium ( $>$ Fig.4b and $>$ Fig. 6). WOS: this was seen in the metaplastic mucosa but not in the non-metaplastic mucosa. In the ultra-magnifying NBI images, the WOS was seen as whitish matter within the cellular structure ( $\triangleright$ Fig.5b). Besides these findings, in the metaplastic mucosa, each cellular component was often cloudier than that in the non-metaplastic mucosa ( Fig.4b). Therefore, subepithelial capillaries looked hazy in the metaplastic mucosa compared with the non-metaplastic mucosa. In some areas at the periphery of the metaplastic mucosa, there was a distinctive boundary with the non-metaplastic mucosa ( $\mathbf{F i g} .6$ ).

Prevalence of each finding in metaplastic and non-metaplastic mucosa, its diagnostic values, and interobserver variability were listed in > Table 2. Because all diagnostic values of heterogeneous cellular structure and rough contours of the marginal 

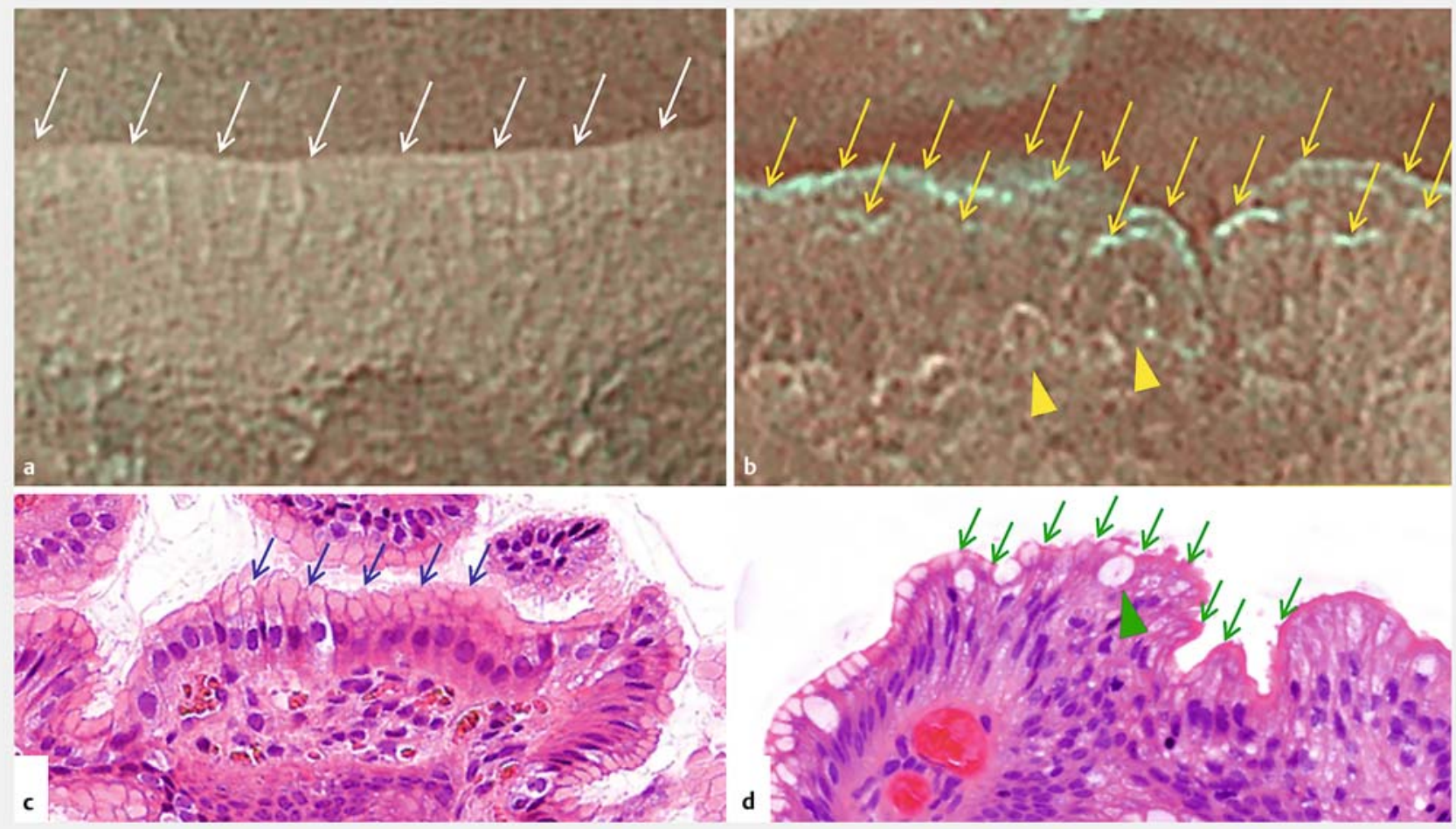

- Fig. 7 a Enlarged image of the marginal crypt epithelium in the non-metaplastic mucosa, showing homogeneous round cellar structure and smooth contour (white arrows). b Image of the marginal crypt epithelium in the metaplastic mucosa, showing heterogeneous cellular structure including sporadic large cells (yellow triangle) and rough multiple contours (yellow arrows). Images $\mathbf{a}$ and $\mathbf{b}$ correspond to the white and yellow rectangles in $\mathbf{~ F i g . ~ 6 , ~ r e s p e c t i v e l y . ~ H i s t o l o g i c a l ~ a p p e a r a n c e ~ o f ~ b i o p s y ~ s p e c i m e n s ~ f r o m ~ a ~ n o n - m e t a p l a s t i c ~ m u c o s a ~ a n d ~} \mathbf{d}$ metaplastic mucosa. The former shows round and homogeneous arrangement of epithelial cells containing uniform mucin, with c smooth epithelial surface (blue arrows). The latter shows heterogeneous epithelial cells, including goblet cells (green triangle), and the $\mathbf{d}$ epithelial surface looked rough (green arrows).

crypt epithelium exceeded $80 \%$, they were regarded as promising diagnostic criteria for GIM in ultra-magnifying NBI.

\section{Histological findings of the metaplastic and non-metaplastic mucosa}

In two patients without endoscopic finding of GIM and three patients with endoscopic finding of GIM, ultra-magnifying NBI images of the non-metaplastic and metaplastic mucosae were captured from the biopsy sites. The surface and intraepithelial structure of the marginal crypt epithelium was compared between the non-metaplastic and metaplastic mucosa. The edge of the surface epithelium in the non-metaplastic mucosa was

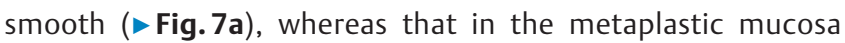
was uneven and somewhat jagged ( $\mathbf{F i g . 7 b}$ ). This suggests that these histological findings presented as rough contours of the marginal crypt epithelium in the ultra-magnifying NBI images ( $\triangleright$ Fig.7c and $\triangleright$ Fig.7d). For the intraepithelial structure, epithelial cells and intracellular distribution of mucin were uniform in the surface epithelium in the non-metaplastic mucosa ( Fig. 7a), whereas those in the metaplastic mucosa were nonuniform, and there were sporadic, large goblet cells ( $\triangleright$ Fig. $\mathbf{7 b}$ ). This suggests that these findings presented as heterogeneous cellular structures with sporadic distribution of large cells in the ultra-magnifying NBI images ( $\mathbf{F i g . 7 c}$ and $\triangleright$ Fig. 7d).

\section{Discussion}

In our study, non-contact ultra-magnifying NBI using endocytoscopy showed cellular structure in the gastric epithelium, and characteristic findings of GIM, heterogeneous cellular structure including sporadic large cells, and rough contours of the marginal crypt epithelium with LBCs. To the best of our knowledge, this is the first study to investigate ultrastructure of the epithelium of the gastric mucosa in vivo.

Originally, the endocytoscopy system was designed for contact observation of the mucosa. Therefore, in conventional endocytoscopy, the objective lens makes contact with the mucosal surface and the superficial mucosal images are observed with backward scattering light through the mucosa. For instance, absorptive dyes are used to stain nuclei [13-15], and sometimes cytoplasm to contrast nuclear images [16-19], and the nuclear images are observed as a silhouette. In this method, nuclear findings are evaluated for diagnosis, but other findings of the epithelium are not assessable. Moreover, conventional contact endocytoscopy requires time and effort to remove mucus from the mucosal surface, and to stain nuclei and cytoplasm with application of dyes to the mucosal surface. To the best of our knowledge, this is the first report to indicate the 
- Table2 Diagnostic values for ultra-magnifying NBI findings from the gastric mucosa.

\begin{tabular}{|c|c|c|c|c|c|c|}
\hline & $\begin{array}{l}\text { Metaplastic } \\
\text { mucosa }(n=28)\end{array}$ & $\begin{array}{l}\text { Non-metaplastic } \\
\text { mucosa }(n=32)\end{array}$ & $\begin{array}{l}\text { Sensitivity \% } \\
(95 \% \mathrm{Cl})\end{array}$ & $\begin{array}{l}\text { Specificity \% } \\
(95 \% \mathrm{CI})\end{array}$ & $\begin{array}{l}\text { Accuracy \% } \\
(95 \% \mathrm{Cl})\end{array}$ & $\begin{array}{l}\text { Kappa } \\
\text { value }\end{array}$ \\
\hline $\begin{array}{l}\text { Heterogeneous cellular } \\
\text { structure }\end{array}$ & 23 & 2 & $82(68-96)$ & $94(85-100)$ & $88(80-96)$ & 0.70 \\
\hline $\begin{array}{l}\text { Rough contour of the } \\
\text { marginal crypt epithelium }\end{array}$ & 24 & 2 & $86(73-99)$ & $94(85-100)$ & $90(82-98)$ & 0.71 \\
\hline Light blue crest & 22 & 2 & $79(63-94)$ & $94(85-100)$ & $87(78-95)$ & 0.87 \\
\hline White opaque substance & 15 & 3 & $54(35-72)$ & $91(81-100)$ & $73(62-85)$ & 0.64 \\
\hline
\end{tabular}

possible use of ultra-magnifying NBI to evaluate ultrastructure of the epithelium without any staining.

Some investigators have used contact ultra-magnifying NBI to diagnose diminutive colorectal polyps [20], and invasive colorectal carcinoma [21,22]. However, in contact observation, $\mathrm{NBI}$ images are dark and coarse because the NBI light is dimmer than white light and the short wavelength light does not penetrate the mucosa well. Therefore, only vascular findings are evaluated. In our non-contact observation, NBI light illuminated the mucosal surface and precise ultra-magnifying reflected images were captured. We found that the marginal crypt epithelium of the metaplastic mucosa had rough and multiple contours with LBCs. Scanning electron microscopy showed that the mucosal surface of GIM was undulated [23] and when we observed the structure tangentially, it appeared to have multiple contours. Such three-dimensional aspects of the mucosal surface may not be recognized by histological sections. Ultra-magnifying NBI facilitates understanding the ultrastructure of the surface epithelium in the gastrointestinal tract.

Beyond a certain magnification level of the ultra-magnifying NBI images, we found that the cellular structure inside the marginal crypt epithelium could be seen. The cellular structure of the non-metaplastic mucosa was round and homogeneous. In relation to the size of each component of the cellular structure and width of the marginal crypt epithelium, we speculated that the size of the cellular components in the ultra-magnifying NBI images corresponded to that of the cell. Moreover, scanning electron microscopy shows that the surface of the non-metaplastic gastric epithelium has a cobblestone-like appearance that consists of apices of surface mucous cells and intercellular clefts [23-25]. This is similar to the findings of ultra-magnifying $\mathrm{NBI}$. When a semi-transparent substance is illuminated, its components are visualized according light reflection at the border between substances with different refractive indices. Therefore, we suspected that each component of the cellular structure in the ultra-magnifying NBI images corresponded with mucin in the cell. Histological analysis of biopsy specimens showed that the structure of the cells and mucin in the nonmetaplastic mucosa was round and homogeneous, whereas that in the metaplastic mucosa was heterogeneous. We did not confirm that the small cellular components in the ultra- magnifying NBI images were identical to the real cells; therefore, the term cellular structure was used in this study. Further analysis is needed for histological findings that correspond to ultra-magnifying NBI images.

In ultra-magnifying methylene blue chromoendoscopy using endocytoscopy, goblet cells are seen as small unstained circular areas in the marginal crypt epithelium [18], showing sensitivity of $89 \%$ and specificity of $71 \%$ for diagnosis of GIM [26]. In ultra-magnifying NBI, sporadic distribution of large cells is consistent with the small unstained circular areas in ultra-magnifying chromoendoscopy. In the current analysis, sensitivity of this finding was $61 \%$ (95\% Cl 41\%-79\%), which was lower than that of ultra-magnifying chromoendoscopy. One reason for the low sensitivity of ultra-magnifying NBI is difficulty in recognition of the sporadic large cells compared to recognition of unstained areas among the stained epithelium. Generally, color contrast in chromoendoscopic images is higher than that in NBI images.

It is considered that WOS is visualized by strong scattering and reflection of light by the lipid droplets that are absorbed into the mucosa of GIM [27]. In histological examination of mucosa with WOS, lipid droplets are present inside the epithelial

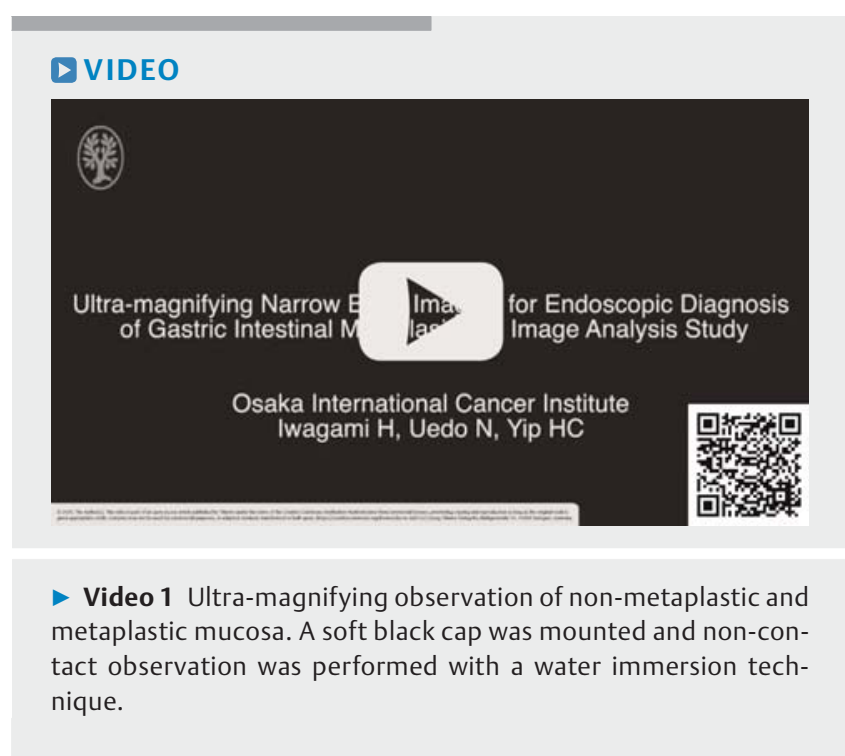


cells in all cases and, in $61.5 \%$ of cases, lipid droplets are also distributed underneath the epithelial cells [26]. In ultra-magnifying NBI, most WOS was observed inside the cellular structure partitioned by intercellular septa. Accordingly, we speculate that intraepithelial, rather than subepithelial, lipid droplets contributed to visualization of the WOS.

Although ultra-magnifying NBI may improve endoscopic diagnosis of GIM, the clinical importance of this method should be investigated further. One of the objectives of diagnosis of GIM is risk assessment for developing gastric cancer. Endoscopic diagnosis of GIM has advantages over biopsy because it avoids risk of bleeding caused by multiple forceps biopsies, and it can quantify the extent of GIM in the gastric mucosa. The utility of near-focus NBI [6, 7] or magnifying NBI [28] for risk staging of gastric cancer has recently been reported. Compared to those methods, non-contact ultra-magnifying observation is technically more demanding. It requires delicate maneuver of the lever to control the magnification and adjust the focus of the endoscopic images. Moreover, because light distribution of the current endocytoscope is not optimized for non-contact observation, an endoscopist has to adapt working distance between lens and the mucosa for appropriate illumination with fine push-pull movement of the scope. Furthermore, endocytoscopy is not readily available worldwide. Accordingly, the clinical impact of ultra-magnifying NBI on risk assessment of gastric cancer may not be as high as with conventional observation methods of NBI. We suspect that this method would be useful to reveal the ultrastructure of the gastrointestinal tract and explain the pathogenesis of the disease. Nevertheless, further advancement of technology to capture stable ultra-high magnification image is expected.

We acknowledge two major limitations of this study. At first, we did not take biopsy specimens from all patients and evaluate the histological findings. We used conventional magnifying NBI as a reference standard instead of histology because precise correlation between ultra-magnifying images and histological findings of biopsy specimens is difficult, even with targeted biopsy. However, in endoscopic observation, we can continuously increase magnification and correlate conventional magnifying with ultra-magnifying NB images. Conventional magnifying NBI has high diagnostic accuracy for diagnosis of histological GIM (90\% sensitivity and $90 \%$ specificity) [29]. Next, only representative good images were chosen for analysis, resulting in non-negligible overestimation of diagnostic accuracy. The diagnostic performance of ultra-magnifying NBI using the characteristic endoscopic findings revealed in this study should be investigated in a future prospective study in relation to histological findings.

\section{Conclusion}

In conclusion, analysis of ultra-magnifying NBI of GIM revealed the characteristic epithelial ultrastructure of GIM, heterogeneous cellular structure, and rough contours of the marginal crypt epithelium with LBC. Further investigation of actual diagnostic accuracy and clinical relevance of these endoscopic findings for GIM is warranted.

\section{Acknowledgments}

The authors thank Cathel Kerr, BSc, PhD, from Edanz Group (https://en-author-services.edanzgroup.com/) for editing a draft of this manuscript.

\section{Competing interests}

The authors declare that they have no conflict of interest.

References

[1] Lage J, Uedo N, Dinis-Ribeiro M et al. Surveillance of patients with gastric precancerous conditions. Best Pract Res Clin Gastroenterol 2016; 30: 913-922

[2] Pimentel-Nunes P, Libânio D, Marcos-Pinto R et al. Management of epithelial precancerous conditions and lesions in the stomach (MAPS II): European Society of Gastrointestinal Endoscopy (ESGE), European Helicobacter and Microbiota Study Group (EHMSG), European Society of Pathology (ESP), and Sociedade Portuguesa de Endoscopia Digestiva (SPED) guideline update 2019. Endoscopy 2019; 51: 365-388

[3] Dixon MF, Genta RM, Yardley JH et al. Classification and grading of gastritis. The updated Sydney System. International Workshop on the Histopathology of Gastritis, Houston 1994. Am J Surg Pathol 1996; 20: 1161-1181

[4] Capelle LG, de Vries AC, Haringsma J et al. The staging of gastritis with the OLGA system by using intestinal metaplasia as an accurate alternative for atrophic gastritis. Gastrointest Endosc 2010; 71: 11501158

[5] Uedo $\mathrm{N}$, Ishihara $\mathrm{R}$, lishi $\mathrm{H}$ et al. A new method of diagnosing gastric intestinal metaplasia: narrow-band imaging with magnifying endoscopy. Endoscopy 2006; 38: 819-824

[6] Pimentel-Nunes P, Libânio D, Lage J et al. A multicenter prospective study of the real-time use of narrow-band imaging in the diagnosis of premalignant gastric conditions and lesions. Endoscopy 2016; 48: 723-730

[7] Esposito G, Pimentel-Nunes P, Angeletti S et al. Endoscopic grading of gastric intestinal metaplasia (EGGIM): a multicenter validation study. Endoscopy 2019; 51: 515-521

[8] Inoue H, Sasajima K, Kaga M et al. Endoscopic in vivo evaluation of tissue atypia in the esophagus using a newly designed integrated endocytoscope: a pilot trial. Endoscopy 2006; 38: 891-895

[9] Yao K, Nagahama T, Matsui T. Magnification endoscopy technique based on gastric microvascular architecture. Gastroenterological Endosc 2008; 50: 1145-1153

[10] An JK, Song GA, Kim GH et al. Marginal turbid band and light blue crest, signs observed in magnifying narrow-band imaging endoscopy, are indicative of gastric intestinal metaplasia. BMC Gastroenterol 2012; 12: 169

[11] Kanemitsu T, Yao K, Nagahama T et al. Extending magnifying NBI diagnosis of intestinal metaplasia in the stomach: the white opaque substance marker. Endoscopy 2017; 49: 529-535

[12] Landis JR, Koch GG. The measurement of observer agreement for categorical data. Biometrics 1977; 33: 159-174

[13] Kumagai Y, Monma K, Kawada K. Magnifying chromoendoscopy of the esophagus: in-vivo pathological diagnosis using an endocytoscopy system. Endoscopy 2004; 36: 590-594

[14] Inoue H, Sasajima K, Kaga M et al. Endoscopic in vivo evaluation of tissue atypia in the esophagus using a newly designed integrated endocytoscope: a pilot trial. Endoscopy 2006; 38: 891-895 
[15] Kudo SE, Wakamura K, Ikehara N et al. Diagnosis of colorectal lesions with a novel endocytoscopic classification-a pilot study. Endoscopy 2011; 43: 869-875

[16] Minami H, Inoue H, Yokoyama A et al. Recent advancement of observing living cells in the esophagus using CM double staining: endocytoscopic atypia classification. Dis Esophagus 2012; 25: 235-241

[17] Kodashima S, Fujishiro M, Takubo K et al. Ex-vivo study of high-magnification chromoendoscopy in the gastrointestinal tract to determine the optimal staining conditions for endocytoscopy. Endoscopy 2006; 38: 1115-1121

[18] Ichimasa K, Kudo SE, Mori Y et al. Double staining with crystal violet and methylene blue is appropriate for colonic endocytoscopy: an in vivo prospective pilot study. Dig Endosc 2014; 26: 403-408

[19] Kaise M, Ohkura Y, lizuka T et al. Endocytoscopy is a promising modality with high diagnostic accuracy for gastric cancer. Endoscopy 2015; 47: 19-25

[20] Kataoka S, Kudo SE, Misawa M et al. Endocytoscopy with NBI has the potential to correctly diagnose diminutive colorectal polyps that are difficult to diagnose using conventional NBI. Endosc Int Open 2020; 8: E360-E367

[21] Nakamura H, Kudo SE, Misawa M et al. Evaluation of microvascular findings of deeply invasive colorectal cancer by endocytoscopy with narrow-band imaging. Endosc Int Open 2016; 4: E1280-E1285
[22] Takeda K, Kudo SE, Misawa M et al. Comparison of the endocytoscopic and clinicopathologic features of colorectal neoplasms. Endosc Int Open 2016; 4: E397-E402

[23] Winborn WB, Weser E. Scanning electron microscopy of intestinal metaplasia of the human stomach. Gastrointest Endosc 1983; 29: 201-207

[24] Takagi T, Takebayashi S, Tokuyasu K et al. Scanning electron microscopy on the human gastric mucosa; fetal, normal and various pathological conditions. Acta Pathol Jpn 1974; 24: 233-247

[25] Fallah E, Schuman BM, Watson JH et al. Scanning electron microscopy of gastroscopic biopsies. Gastrointest Endosc 1976; 22: 137-144

[26] Chiu PW, Ng EK, To KF et al. Recognition of goblet cells upon endocytoscopy indicates the presence of gastric intestinal metaplasia. Dig Endosc 2014; 26: 52-56

[27] Yao K, Iwashita A, Nambu M et al. Nature of white opaque substance in gastric epithelial neoplasia as visualized by magnifying endoscopy with narrow-band imaging. Dig Endosc 2012; 24: 419-425

[28] Saka A, Yagi K, Nimura S. OLGA- and OLGIM-based staging of gastritis using narrow-band imaging magnifying endoscopy. Dig Endosc 2015; 27: 734-741

[29] Wang L, Huang W, Du J et al. Diagnostic yield of the light blue crest sign in gastric intestinal metaplasia: a meta-analysis. PLoS One 2014; 9: e92874 